\title{
A case of an aortic dissection in a young adult: a refresher of the literature of this "great masquerader"
}

This article was published in the following Dove Press journal:

International Journal of General Medicine

2I December 20II

Number of times this article has been viewed

\author{
Jérôme Pineault ${ }^{1}$ \\ Denis Ouimet ${ }^{2}$ \\ Vincent Pichette ${ }^{2}$ \\ Michel Vallée ${ }^{2}$ \\ 'Department of Internal Medicine, \\ 2Department of Nephrology, \\ Maisonneuve-Rosemont Hospital, \\ Montreal, Canada
}

\begin{abstract}
Aortic dissection is often misdiagnosed, especially among young patients, and it is associated with a high mortality rate. We present here a case of fatal acute aortic dissection in a young man who was misdiagnosed with pericarditis. We reviewed the literature of acute aortic dissection in young people and we focused particularly on clinical presentations, outcomes and investigations of aortic dissection. We report a case of a 33-year-old man with a history of uncontrolled hypertension with acute pleuretic chest pain who was transferred to our hospital for suspected pulmonary embolism and died of acute hemorragic pericardial effusion from an ascendant aortic dissection. We should never rule out aortic dissection off our differential diagnosis on the sole basis of a patient's young age.

Keywords: pleuritic chest pain, hypertension, aortic dissection
\end{abstract}

\section{Case presentation}

A 33-year-old black man was transferred to our hospital with acute chest pain, and suspicion of pulmonary embolism. He had no family history of cardiovascular disease. His past medical history revealed severe uncontrolled essential hypertension associated with noncompliance with medication and appointments, severe left ventricular hypertrophy $(\mathrm{LVH})$, hypertensive nephrosclerosis with estimated glomerular filtration rate of $35 \mathrm{~mL} / \mathrm{min} / 1.73 \mathrm{~m}^{2}$, mild ascending aorta ectasia (up to $4.2 \mathrm{~cm}$ ), sickle cell trait, and appendicectomy. He had no history of smoking or cocaine abuse. He presented with moderately severe chest and upper abdominal pain radiating to the back, which had begun acutely a few hours before consulting. The pain was pleuritic on the right side of the chest and associated with mild shortness of breath.

On physical examination, temperature and blood oxygen saturation were normal. Pulse and respiratory rates were 80/minute and 18/minute, respectively. Blood pressure was $172 / 85 \mathrm{mmHg}$ in left arm and $176 / 89 \mathrm{mmHg}$ on the right side. These readings were remarkably better than previous ones which were around 200-230/110-120 mmHg. There was no jugular venous distension. Heart rhythm was regular with a II/VI diastolic murmur best heard at the lower right parasternal border. His lungs were clear. He had mild discomfort when palpating his upper abdomen, but it was soft without defense. The rest of the exam was unremarkable.

Lab results showed normal cardiac enzymes, slightly elevated white blood cell count, stable creatinine $(290 \mu \mathrm{mol} / \mathrm{L}$ or $3.28 \mathrm{mg} / \mathrm{dL})$ and marked positive D-dimer (4671 ng/mL). EKG showed signs of left ventricular hypertrophy ( $\mathrm{LVH}$ ) and nonspecific ST-T wave changes. The first chest X-ray was read as normal by the radiologist except for cardiomegaly (Figure 1). Pulmonary embolism was the initial working diagnosis
Correspondence: Michel Vallée

54I5 Assomption Blvd, Montreal,

Quebec, HIT 2M4, Canada

Tel + I 5I4-252-3489

Fax + | 5|4-255-3026

Email mvallee.hmr@ssss.gouv.qc.ca 


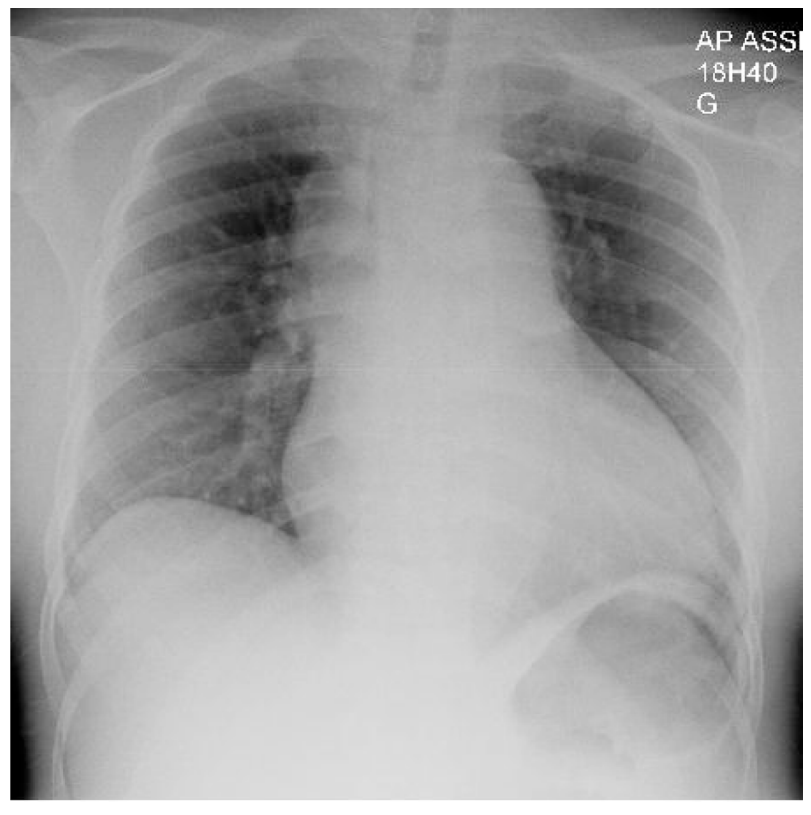

Figure I Antero-posterior chest X-ray of our patient at his initial presentation.

and a contrast computed tomography (CT) scan was ordered but then cancelled because of the risk of contrast-induced nephropathy. Instead, a ventilation-perfusion pulmonary scintigraphy was performed and was negative for pulmonary embolism. Lower limb venous doppler echography was normal. The abdominal ultrasonography was normal.

On the second day in the hospital, a transthoracic echocardiography revealed severe left ventricular hypertrophy, normal left and right ventricular kinetics, mild to moderate aortic regurgitation, and no pericardial effusion. Because of the upper abdominal pain, he was scheduled for an upper gastrointestinal tract endoscopy to rule out peptic ulcer disease.

Three days went by without significant change. Symptoms were moderately severe but had not abated. The endoscopy was normal. On the fifth day of hospitalization, the patient started to present a pericardial friction rub. A diagnosis of pericarditis was retained, possibly of uremic origin. A few hours later, the patient had increasing shortness of breath and a second chest X-ray showed mild bilateral pleural effusions (Figure 2). That same day, the patient presented sudden death with unsuccessful reanimation. The autopsy revealed a massive cardiac hemorragic tamponade from a ruptured retrograde aortic dissection that extended down to the iliac arteries and severe LVH. These findings suggested acute retrograde dissection and rupture in the pericardial space complicating a chronic antegrade aortic dissection associated with severe hypertension. The patient did not have any connective tissue disease or Marfan syndrome.

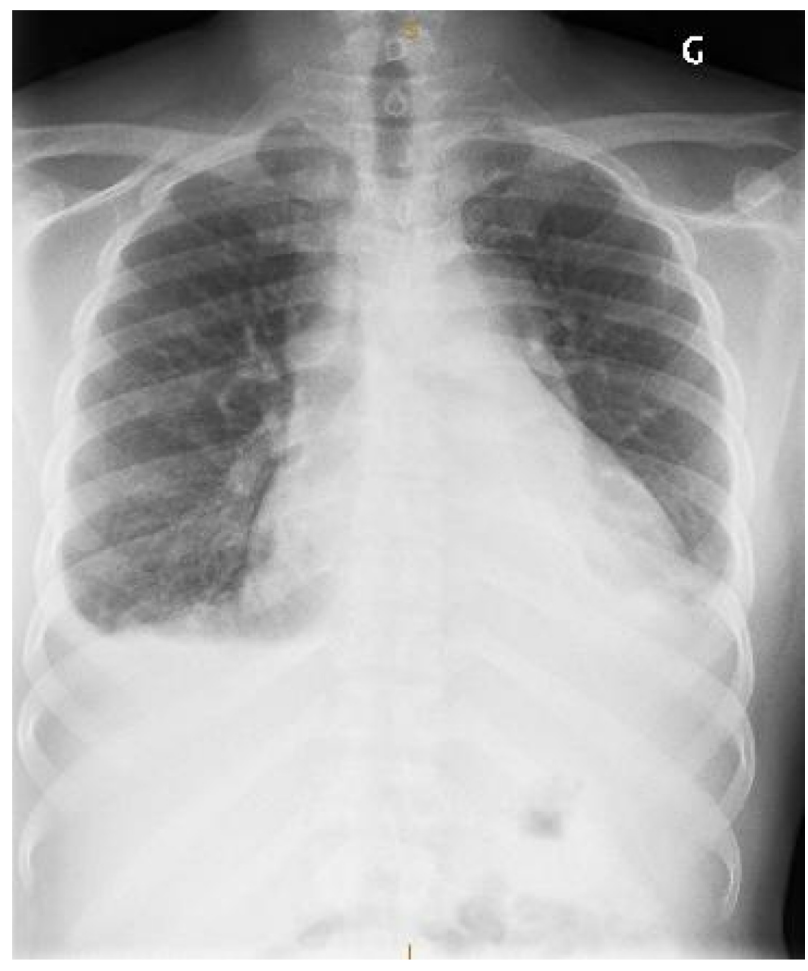

Figure 2 Antero-posterior chest X-ray of our patient 5 days after presentation.

\section{Discussion}

We all know that aortic dissection (AoD) is by far the most common and the most serious condition of the aorta. The associated mortality rate is about $1 \%-3 \%$ per hour with $20 \%-30 \%$ of deaths occurring in the first 24 hours and roughly $80 \%$ in 2 weeks. ${ }^{1}$ Although it is very uncommon with an incidence estimated from 5 to 30 cases per million population yearly in the United States according to the International Registry of Aortic Dissection (IRAD), compared with an estimated 4400 cases per million yearly for acute myocardial infarction, it remains a critical medical condition that necessitates prompt diagnosis for proper life-saving treatment. ${ }^{2}$ Unfortunately diagnosis is not uncommonly delayed or missed with lethal consequences.

\section{Clinical presentations and associated conditions}

AoD can present with a wide variety of signs and symptoms. Chest pain is by far the most common presenting symptom, being present in $75 \%$ of patients. In $85 \%$ of patients, the pain is abrupt and severe. ${ }^{2,3}$ The classical description of "tearing pain" is only present for $51 \%$ of patients; most describe it as sharp or stabbing. ${ }^{3}$ However, $5 \%-15 \%$ of patients get to the emergency department without any pain. ${ }^{2-4}$ Other clinical presentations include hypertension $(32 \%-49 \%)$, aortic 
regurgitation $(32 \%-45 \%)$, pulse deficit or limb ischemia (15\%-26\%), syncope ( $9 \%-13 \%)$, shock and/or tamponade $(8 \%-18 \%)$, hypotension without shock $(8 \%-14 \%)$, heart failure - usually due to acute aortic regurgitation $(6 \%)$, focal neurological deficits $(5 \%-8 \%)$ and pericardial friction rub $(2 \%)^{2,3}$ Even fever of unknown origin has been described as the initial presentation of an AoD. ${ }^{5}$ Diagnosis is missed at presentation in up to $38 \%$ of patients and found at postmortem (without any prior known or suspected cause of death) in $28 \%$ of them. ${ }^{2,4}$ Conditions associated with AoD are hypertension $(72 \%)$, male sex $(65 \%)$, older age (mean age: 63 years old), atherosclerosis (31\%), known aortic aneurysm and previous cardiovascular surgery or aortic dissection ( $20 \%)$, Marfan syndrome (5\%), vascular Ehlers-Danlos syndrome, LoeysDietz syndrome, Turner syndrome, bicuspid aortic valve, familial form of aortic aneurysm/dissection, large vessel vasculitis, pregnancy, and cocaine use $(<1 \%)$. $2,3,6$

\section{Diagnosing AoD}

Recognizing this condition may be difficult, but clinicians can count on several tools to help them diagnose AoD. Some are easily available, such as chest X-ray and Electrocardiogram (EKG), but their specificity and sensitivity are very low. Again, according to the IRAD and the American Heart Association (AHA) guidelines, a chest X-ray was ordered in $100 \%$ of patients who were finally diagnosed with an AoD, but $10 \%-12 \%$ were normal and only $60 \%$ had the characteristic "mediastinal widening" (Figure 3)., Some nonspecific findings like abnormal aortic contour (50\%) and abnormal cardiac contour (26\%) may be noticeable on X-rays. ${ }^{2}$ Thirty-one percent of EKGs were normal, $41 \%$ had nonspecific ST-T changes, $26 \%$ had left ventricular hypertrophy, and 15\% showed signs of ischemia. ${ }^{2}$ Table 1 shows the imaging modalities that can be used to diagnose AoD, with their reported sensitivity and specificity and Appendix 1 shows the diagnostic algorithm (adapted version) for AoD from the AHA 2010 guidelines. ${ }^{2,3,7,8}$ CT scanning with IV contrast is the most frequent imaging modality used because of its availability, its lower reliance on operator skills, and its current improved accuracy and shorter acquisition time compared with the older CT scans, with good sensitivity and specificity. ${ }^{7}$ Transthoracic echocardiography shows variable sensitivity possibly owing to operator skills. Transesophageal echo shows very good sensitivity and specificity when available and does not require contrast agent. Magnetic resonance imaging appears to be the best imaging study for AoD, but is not widely available, needs a trained radiologist to interpret the images, and image acquisition is slower than in other diagnostic methods. Aortography was once
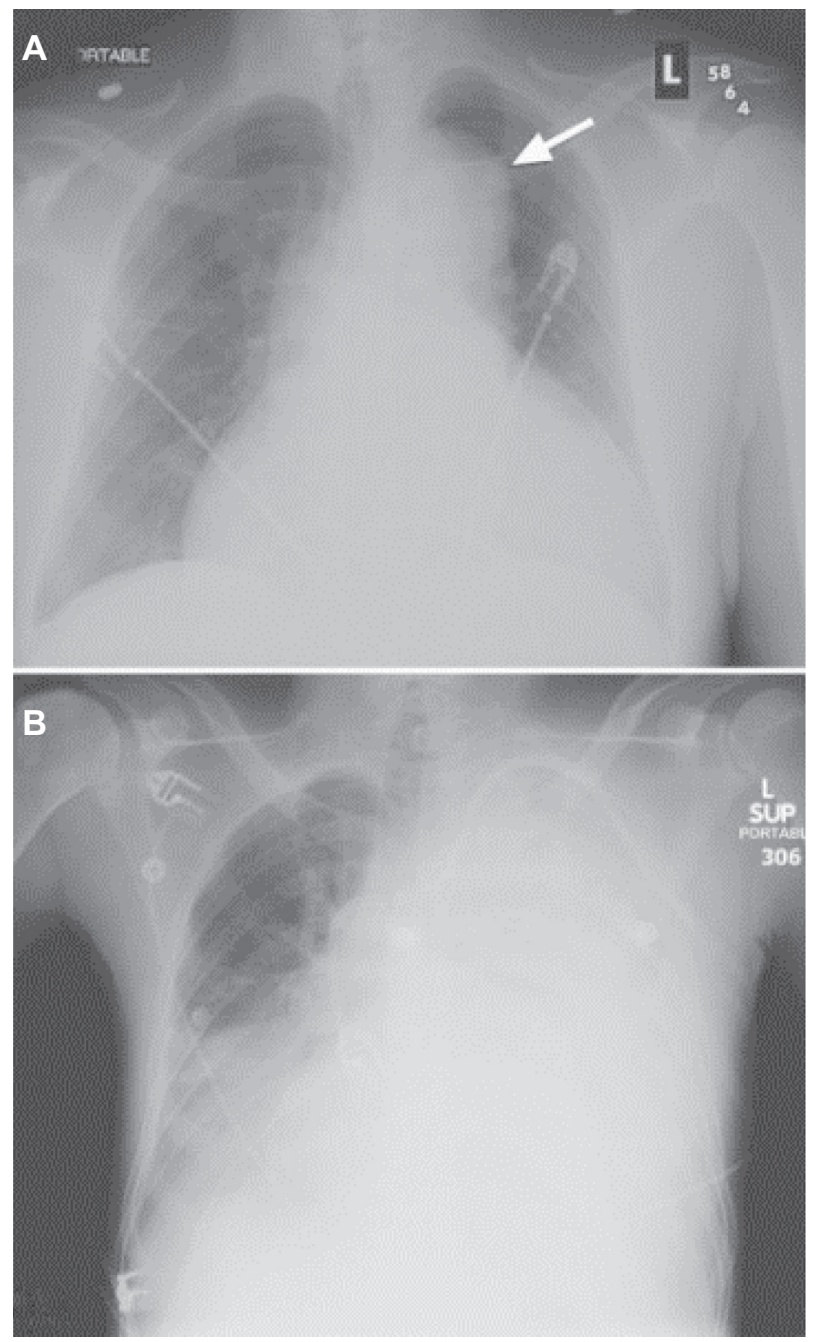

Figure 3 Chest $X$-ray of a patient with diagnosed AoD. (A) Widened mediastinum, abnormal aortic contour and enlarged cardiac silhouette. (B) Acute left hemothorax after ruptured AoD dissection.

This figure was published in Braunwald's heart disease - a textbook of cardiovascular medicine, 9th ed. Bonow RO, Mann DL, Zipes DP, Libby P. Reproduced with permission, Copyright Elsevier (20I2).

the gold standard imaging modality, but its invasiveness now makes it obsolete except in special circumstances.

\section{Findings in young adults with AoD}

AoD in young adults remains an unexpected cardiovascular catastrophe. According to IRAD study results, only $7 \%$ of all

Table I Reported sensitivity and specificity of the different imaging modalities used to diagnose AoD

\begin{tabular}{lll}
\hline Imaging modality & Sensitivity & Specificity \\
\hline CT scanning with contrast & $83 \%-94 \%$ & $87 \%-100 \%$ \\
TTE & $59 \%-80 \%$ & $83 \%-96 \%$ \\
TOE & $98 \%$ & $95 \%$ \\
MRI with gadolinium & $95 \%-100 \%$ & $95 \%-100 \%$ \\
Aortography & $87 \%$ & $85 \%$ \\
\hline
\end{tabular}

Abbreviations: $\mathrm{CT}$, computed tomography; MRI, magnetic resonance imaging; TOE, transesophageal echo; TTE, transthoracic echocardiography. 
AoD occurred in people $<40$ years of age. ${ }^{9}$ Of these, $50 \%$ had Marfan syndrome (compared with only $2 \%$ of the $>40$ years cohort), $9 \%$ had bicuspid aortic valve ( $1 \%$ in the $>40$ years cohort), $12 \%$ had prior aortic valve replacement, and $3 \%$ were women in the peripartum period. ${ }^{9}$ These risk factors were significantly more frequent than in the older age group. ${ }^{9}$ However, clinical presentations were similar in both groups, pain, blood pressure variation (high or low) and diastolic murmur being the most common clinical findings. The only noticeable difference is that older people experienced more hypertensive episodes than young people. ${ }^{9}$ Surprisingly, there is no difference in survival rates between the two groups. Mortality rate was $22 \%$ in young patients and $24 \%$ in older patients. ${ }^{9}$ Tamponade (15\% vs $12 \%$ ), myocardial ischemia/ infarction ( $6 \%$ vs 9\%) and acute kidney injury (13\% vs $18 \%$ ) were among the other complications of AoD, without any significant difference between the two groups. ${ }^{9}$

An interesting finding of the IRAD database is that younger people were much more likely to have prior aortic dilation, without any known connective tissue disease. ${ }^{9}$ The measurements of several aortic parts showed a significantly more frequent dilation of these portions of the aorta prior to dissection, than in the older group. ${ }^{9}$ Thus we should include AoD in our differential diagnosis of young patients with chest pain and known aortic dilation (even mild).

\section{D-dimer testing and AoD}

An interesting concept is emerging in the approach to suspected AoD and the use of D-dimer testing as a screening test for AoD. The use of D-dimer testing is well known for excluding pulmonary embolism due to its high sensitivity, and every physician knows that elevated D-dimers should raise at least a mild suspicion for pulmonary embolism. ${ }^{10}$ Some studies have been published on the use of D-dimer testing to rule out AoD. Reported sensitivity of D-dimer testing in the setting of suspected AoD is $100 \%$ and its degree of elevation even showed a trend toward extension of the dissection but not with the outcomes. ${ }^{11,12}$ Even if it is not in the actual guidelines of AoD diagnostic algorithm, the use of D-dimer testing seems to be a good and reliable marker to rule out AoD in our differential diagnosis for patients presenting with acute chest pain. Further investigations with larger study populations should be done to assess the real utility of this biomarker, in ruling out this morbid condition.

\section{Conclusion}

We should always keep in mind that AoD is a possible diagnosis even in the young population. Although it is not frequent, assessing risk factors and high risk clinical features for that condition should be done promptly in a patient complaining of acute chest or abdominal pain in order to make the proper diagnostic exam, because of fatalities associated with that condition if not treated quickly. Maybe D-dimer testing could be an interesting biomarker to rapidly and easily rule out this potentially fatal condition.

\section{Disclosure}

The authors report no conflicts of interest in this work.

\section{References}

1. Meszaros I. Epidemiology and clinicopathology of aortic dissection. Chest. 2000;117(5):1271-1278.

2. Hagan PG, Nienaber CA, Isselbacher EM, et al. The International Registry of Acute Aortic Dissection (IRAD): new insights into an old disease. JAMA. 2000;283:897-903.

3. Hiratzka LF. ACCF/AHA Guidelines for the diagnosis and management of patients with thoracic aortic disease. Circulation. 2010: e266-e348.

4. Spittell PC, Spittell JA Jr, Joyce JW, et al. Clinical features and differential diagnosis of aortic dissection: experience with 236 cases (1980 through 1990). Mayo Clin Proc. 1993;68:642-651.

5. McKeown PP, Campbell NP. Pyrexia of unknown origin and aortic dissection. Int $J$ of Cardiol. 1989;25:124-126.

6. Rushid J, Eisenberg MJ, Topol EJ. Cocaine-induced aortic dissection. Am Heart J. 1996;132:1301-1304.

7. Matalanis G. Acute aortic dissection: how to confirm the diagnosis? Heart Lung Circ. 2004;13:127-128.

8. Petasnick JP. Radiologic evaluation of aortic dissection. Radiology. 1991; 180:297-305.

9. Januzzi JL, Isselbacher EM, Fattori R, et al. Characterizing the young patient with aortic dissection: results from the International Registry of Aortic Dissection (IRAD). J Am Coll Cardiol. 2004;43:665-669.

10. Goldhaber SZ, Simons CR, Elliott CG, et al. Quantitative plasma D-dimer levels among patients undergoing pulmonary angiography for suspected pulmonary embolism. JAMA. 1993;270:2819-2822.

11. Weber T, Högler S, Auer J, et al. D-dimer in acute aortic dissection. Chest. 2003;123:1375-1378.

12. Eggebrecht H, Naber CK, Bruch C, et al. Value of plasma fibrin D-dimers for detection of acute aortic dissection. J Am Coll Cardiol. 2004;44:804-809. 


\section{Appendix}

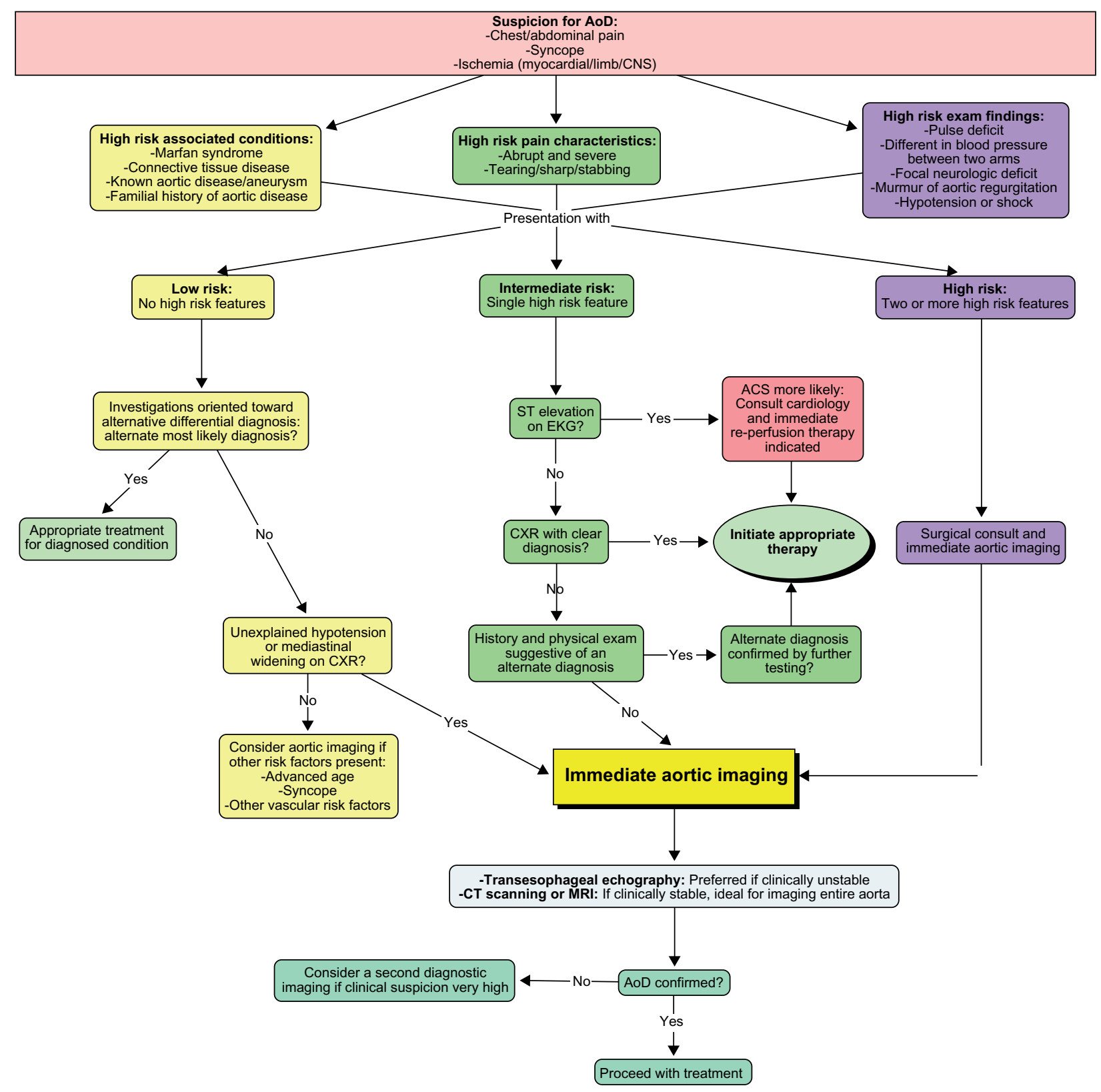

Appendix I AoD diagnostic algorithm.

Reprinted with Permission, Circulation. 2010;121:e266-e369 @2010 American Heart Association, Inc. For the official algorithm, please see Hiratzka. ${ }^{3}$

Abbreviations: ACS, acute coronary syndrome; AoD, aortic dissection; CT, computed tomography; MRI, magnetic resonance imaging; CXR, chest X-ray.

International Journal of General Medicine

Dovepress

\section{Publish your work in this journal}

The International Journal of General Medicine is an international, peer-reviewed open-access journal that focuses on general and internal medicine, pathogenesis, epidemiology, diagnosis, monitoring and treatment protocols. The journal is characterized by the rapid reporting of reviews, original research and clinical studies across all disease areas.
A key focus is the elucidation of disease processes and management protocols resulting in improved outcomes for the patient. The manuscript management system is completely online and includes a very quick and fair peer-review system. Visit http://www.dovepress.com/ testimonials.php to read real quotes from published authors.

Submit your manuscript here: http://www.dovepress.com/international-journal-of-general-medicine-journal 